Journal Club

Editor's Note: These short, critical reviews of recent papers in the Journal, written exclusively by graduate students or postdoctoral fellows, are intended to summarize the important findings of the paper and provide additional insight and commentary. For more information on the format and purpose of the Journal Club, please see http://www.jneurosci.org/misc/ifa_features.shtml.

\title{
mTOR Signaling in Epileptogenesis: Too Much of a Good Thing?
}

\author{
Ruifeng Cao, ${ }^{1,2}$ Aiqing Li, ${ }^{1}$ and Hee-Yeon Cho ${ }^{1,3}$ \\ ${ }^{1}$ Department of Neuroscience, ${ }^{2}$ Neuroscience Graduate Studies Program, and ${ }^{3}$ Molecular, Cellular, Developmental Biology Graduate Program, Ohio State \\ University, Columbus, Ohio 43210 \\ Review of Zeng et al.
}

Mammalian target of rapamycin (mTOR) is a conserved serine/threonine protein kinase. It forms multiprotein complexes that couple extracellular signals (growth factors, nutrient availability, energy status, and stress) to cellular metabolic resources to balance anabolic and catabolic processes. In the developing CNS, mTOR regulates neuronal survival and differentiation, as well as axon growth and navigation, dendritic arborization, and synaptogenesis. In the adult CNS, mTOR is crucial for many forms of synaptic plasticity, such as long-term potentiation in hippocampus, and thereby plays an important role in the process of learning and memory. In the hypothalamus, mTOR functions as an energy sensor to control animal food intake and regulate body energy balance. It also responds to external light and regulates circadian clock neurons in the suprachiasmatic nuclei. Because of its complex roles in CNS physiology, it is not surprising that disruption of mTOR signaling may contribute to the pathophysiology of various neurological diseases. Indeed, recent studies have revealed that mTOR activity is upregulated or downregulated in brain tumors, tuberous sclerosis complex, cortical dysplasia, traumatic brain injury, and neu-

\footnotetext{
Received July 20, 2009; revised Aug. 21, 2009; accepted Aug. 24, 2009.

We are indebted to Dr. Karl Obrietan for his mentorship and critical reading of this manuscript.

Correspondence should be addressed to Ruifeng Cao, Department of Neuroscience, Ohio State University, 4030 Graves Hall, 333 W 10th Avenue, Columbus, 0H 43210. E-mail: cao.67@osu.edu.

DOI:10.1523/JNEUROSCI.3486-09.2009

Copyright $\odot 2009$ Society for Neuroscience $\quad$ 0270-6474/09/2912372-02\$15.00/0
}

rodegenerative disorders such as Alzheimer's, Parkinson's, and Huntington's diseases (Inoki et al., 2005).

Epilepsy, one of the most common neurological diseases, is characterized by spontaneous recurrent seizures. Epileptogenesis refers to a series of events that occur between the event that causes epilepsy and the first spontaneous seizure. Understanding this process may help to identify molecular targets for drugs that could prevent or treat epilepsy. The cellular alterations involved in epileptogenesis include neuronal cell death, neurogenesis, mossy fiber sprouting and gliosis, etc. However, the molecular mechanisms underlying these events are poorly understood.

Recently, Zeng et al. (2009) suggested a role of mTOR signaling in epileptogenesis. They observed significant effects of mTOR inhibition on several major aspects of epilepsy pathophysiology, including neuronal degeneration, mossy fiber sprouting, neurogenesis, and recurrent seizure.

Zeng et al. (2009) first demonstrated that the mTOR pathway was upregulated by seizure activity biphasically, at $1-24 \mathrm{~h}$ after seizure onset and $3 \mathrm{~d}-5 \mathrm{w}$ after status epilepticus (SE), a state of persistent seizure lasting longer than $30 \mathrm{~min}$. They used kainate, an ionotropic glutamate receptor agonist, to induce seizure in rats. Because of the wide distribution of glutamate receptors in the brain, systemic application of kainate excites multiple brain regions, especially hippocampal CA3 neurons. The kainate-induced firing generates synchro- nized activity (exhibited as SE) in the dense network of recurrent glutamatergic collaterals, and the activity propagates to other limbic structures including dentate gyrus, CA1, and entorhinal cortex. This synchronous activity activates many intracellular signal transduction pathways including mTOR signaling. Notably, kainate did not trigger massive activation of these pathways unless SE occurred: in animals that never developed SE after kainate administration, the kinase activation was minimal. Based on these results, the acute phase of mTOR activation can be induced directly by initial SE-evoked synaptic activity; while the second activation may be a consequence of ensuing spontaneous action potential discharges due to enhancement of synaptic efficacy after the initial episode of SE.

Next, Zeng et al. (2009) demonstrated that although it did not alter the characteristics (latency, duration, and severity) of kainate-induced SE, inhibiting mTOR signaling with rapamycin pretreatment significantly attenuated SE-induced neuronal degeneration. The neuroprotective effect of rapamycin is at first surprising, given that rapamycin is widely used to induce apoptosis of cancer cells. However, as the authors discussed, mTOR has both antiapoptotic and proapoptotic effects (Castedo et al., 2002). Along these lines, there is evidence that mTOR can translocate from the cytoplasm to the nucleus and cause phosphorylation of P53, and transcriptional activation and induction of proapoptotic proteins 
such as BAX, and thereby activate the intrinsic cell death pathway. Thus, rapamycin may inhibit mTOR-mediated apoptotic pathways and decrease cell death.

An alternative mTOR-mediated neuroprotective mechanism may be related to the role of mTOR in autophagy inhibition (Ravikumar et al., 2004). Macroautophagy is a major, evolutionarily conserved response to bioenergetic stresses, which has the capacity to remove aggregated proteins and damaged organelles such as mitochondria. It is a highly regulated catabolic pathway that balances rates of protein induction and degradation to execute its cellular roles (Cherra and Chu, 2008). Growing evidence supports a role of autophagic stress-induced death of neurons in several in vitro and in vivo models of neuron death and neurodegeneration, including the kainate-induced seizure model (Shacka et al., 2007). Rapamycin may target the autophagy pathway during seizure and restore autophagic balance to the intracellular system, allowing neurons to remain functional in the face of rising levels of stress in seizure.

It is also possible that the neuroprotective effects of rapamycin result from its pharmacological effects other than mTOR inhibition. Rapamycin is an immunophilin ligand with neurotrophic activity. Modification of rapamycin at the mTOR binding region (to abolish its binding and inhibition to mTOR) yielded novel immunophilin ligands that had potent neurotrophic activities in cortical neuronal cultures and a rodent ischemic stroke model (Ruan et al., 2008). Electrophysiological analysis indicated that these compounds could inhibit L-type $\mathrm{Ca}^{2+}$ channels in rat hippocampal neurons and dorsal root ganglia/neuroblastoma cells. It is thought that these immunophilin ligands can protect neurons from $\mathrm{Ca}^{2+}$-induced cell death by modulating $\mathrm{Ca}^{2+}$ channels.

Zeng et al. (2009) continued to look at the efficacy of rapamycin on seizureinduced mossy fiber sprouting and neurogenesis, which may be major contributors to epileptogenesis. They found that both neurogenesis and mossy fiber sprouting were significantly decreased by rapamycin. Because both pretreatment (before kainate injection) and post-treatment (after SE) doses of rapamycin were effective in suppressing recurrent seizure, the late phase of $\mathrm{mTOR}$ activation seems to play a more important role in these later stages of epileptogenesis.

Mossy fiber sprouting is a common abnormality in patients with temporal lobe epilepsy. Mossy fiber sprouting creates an aberrant excitatory-feedback network among granule cells. Many studies have indicated a close association between mossy fiber sprouting and spontaneous recurrent seizures. Previous attempts to block mossy fiber sprouting have met with limited success. In the study by Zeng et al. (2009), pretreatment with rapamycin for 3 consecutive days before SE initiation prevented epilepsyinduced mossy fiber sprouting. However, the post-treatment, started $24 \mathrm{~h}$ after SE, required continuous administration of rapamycin to repress the mossy fiber sprouting, indicating its refractory nature.

A paper published more recently in the Journal of Neuroscience also reported effects of mTOR inhibition on seizure-induced mossy fiber sprouting (Buckmaster et al., 2009). In a pilocarpine-induced rat model of epilepsy, rapamycin was chronically infused into the dorsal hippocampus $2 \mathrm{~h}$ after the SE onset. Infusion for 1 month reduced aberrant mossy fiber outgrowth. However, suppression also required continual treatment, and rapamycin treatment did not reverse already established axon reorganization. Together, these results suggest that mTOR is crucial to both initiation and progression of mossy fiber sprouting. The most effective therapy is administration of rapamycin before the induction of SE. Hence, once the SE process is finished, the progression of mossy fiber sprouting is irreversible. While continuous application of rapamycin can inhibit growth, the driving force persists: once rapamycin treatment is stopped, mossy fiber sprouting will reinitiate.

The results of the present study suggest that the role of mTOR in mossy fiber sprouting is comparable to its role in neural development and synaptic plasticity under physiological conditions. Extensive studies have indicated that mTOR-dependent translational control plays a key role in regulating long-term morphological changes in neuronal spines, dendrites, and circuits. mTOR activation leads to phosphorylation of multiple downstream effectors such as the eIF4E-binding proteins (4E-BPs) and S6 kinases and stimulates translation of a subset of mRNAs (Costa-Mattioli et al., 2009). These mTORdependent molecular mechanisms of translational control could also be involved in its role in seizure-induced mossy fiber sprouting.

Recent work from other research groups has also indicated a role of mTOR in mediating neural progenitor/stem cell proliferation. For example, it is reported that Akt-mTOR signaling regulates progenitor cell responses to extrinsic signals. Increased Akt-1 expression in cortical progenitors enhances proliferation and survival in vitro. This process depends on mTOR activity, and rapamycin has been shown to eliminate the Akt-1-mediated increase in stem cells and to reduce proliferation (Sinor and Lillien, 2004). Moreover, Notch ligand can expand stem cells both in vitro and in vivo. This effect is also mediated by mTOR signaling (Androutsellis-Theotokis et al., 2006).

In summary, the work of Zeng et al. (2009) provides evidence that mTOR signaling is involved in the development of epilepsy. Their results should be of interest to clinicians, because rapamycin is an FDA-approved drug. However, more experimental work is needed to clarify the specific molecular mechanisms by which mTOR functions in the multiple aspects of epileptogenesis.

\section{References}

Androutsellis-Theotokis A, Leker RR, Soldner F, Hoeppner DJ, Ravin R, Poser SW, Rueger MA, Bae SK, Kittappa R, McKay RD (2006) Notch signalling regulates stem cell numbers in vitro and in vivo. Nature 442:823-826.

Buckmaster PS, Ingram EA, Wen X (2009) Inhibition of the mammalian target of rapamycin signaling pathway suppresses dentate granule cell axon sprouting in a rodent model of temporal lobe epilepsy. J Neurosci 29:8259-8269.

Castedo M, Ferri KF, Kroemer G (2002) Mammalian target of rapamycin (mTOR): pro- and anti-apoptotic. Cell Death Differ 9:99-100.

Cherra SJ, Chu CT (2008) Autophagy in neuroprotection and neurodegeneration: a question of balance. Future Neurol 3:309-323.

Costa-Mattioli M, Sossin WS, Klann E, Sonenberg N (2009) Translational control of long-lasting synaptic plasticity and memory. Neuron 61:10-26.

Inoki K, Corradetti MN, Guan KL (2005) Dysregulation of the TSC-mTOR pathway in human disease. Nat Genet 37:19-24.

Ravikumar B, Vacher C, Berger Z, Davies JE, Luo S, Oroz LG, Scaravilli F, Easton DF, Duden R, O'Kane CJ, Rubinsztein DC (2004) Inhibition of mTOR induces autophagy and reduces toxicity of polyglutamine expansions in fly and mouse models of Huntington disease. Nat Genet 36:585-595.

Ruan B, Pong K, Jow F, Bowlby M, Crozier RA, Liu D, Liang S, Chen Y, Mercado ML, Feng X, Bennett F, von Schack D, McDonald L, Zaleska MM, Wood A, Reinhart PH, Magolda RL, Skotnicki J, Pangalos MN, Koehn FE, et al. (2008) Binding of rapamycin analogs to calcium channels and FKBP52 contributes to their neuroprotective activities. Proc Natl Acad Sci U S A 105:33-38.

Shacka JJ, Lu J, Xie ZL, Uchiyama Y, Roth KA, Zhang J (2007) Kainic acid induces early and transient autophagic stress in mouse hippocampus. Neurosci Lett 414:57-60.

Sinor AD, Lillien L (2004) Akt-1 expression level regulates CNS precursors. J Neurosci 24:85318541.

Zeng LH, Rensing NR, Wong M (2009) The mammalian target of rapamycin signaling pathway mediates epileptogenesis in a model of temporal lobe epilepsy. J Neurosci 29:6964-6972. 